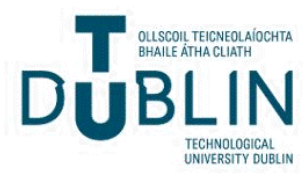

Technological University Dublin

ARROW@TU Dublin

2015-07-16

\section{Design Method for Wideband Circularly Polarized Slot Antennas}

\author{
Adam Narbudowicz \\ Technological University Dublin, adam.narbudowicz@mydit.ie \\ Matthias John \\ Technological University Dublin, matthias.john@tudublin.ie \\ Vit Sipal \\ Technological University Dublin
}

See next page for additional authors

Follow this and additional works at: https://arrow.tudublin.ie/ahfrcart

Part of the Electrical and Electronics Commons, Electromagnetics and Photonics Commons, and the Systems and Communications Commons

\section{Recommended Citation}

Ammann, M.J. et al. (2015) Design Method for Wideband Circularly Polarized Slot Antennas," in Antennas and Propagation, IEEE Transactions on , vol.63, no.10, pp.4271-4279, Oct. 2015 doi:10.1109/

TAP.2015.2456954

This Article is brought to you for free and open access by the Antenna \& High Frequency Research Centre at ARROW@TU Dublin. It has been accepted for inclusion in Articles by an authorized administrator of ARROW@TU Dublin. For more information, please contact arrow.admin@tudublin.ie, aisling.coyne@tudublin.ie, gerard.connolly@tudublin.ie.

Funder: Science Foundation Ireland and Irish Research Council

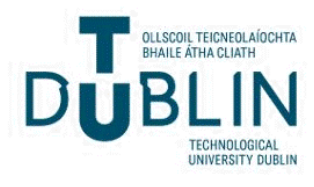


Authors

Adam Narbudowicz, Matthias John, Vit Sipal, Xiulong Bao, and Max Ammann

This article is available at ARROW@TU Dublin: https://arrow.tudublin.ie/ahfrcart/61 


\title{
Design Method for Wideband Circularly Polarized Slot Antennas
}

\author{
Adam Narbudowicz, Member, IEEE, Matthias John, Member, IEEE, Vit Sipal, Member, IEEE, \\ Xiulong Bao, Senior Member, IEEE, and Max J. Ammann, Senior Member, IEEE
}

\begin{abstract}
A compact ultra wideband circularly-polarized slot antenna is proposed with a methodology for automated re-optimization for packaging with proprietary devices. The slot structure employs a dual-feed with an integrated compact ultra wideband phase shifter. The design process involves an advanced optimization algorithm and demonstrates the use of spline curves for generation of circularly polarized signals. These allow easy optimization, while preserving phase coherency across a wide band. The prototyped antenna has a circularly-polarized bandwidth of $54 \%$ (from $3.2 \mathrm{GHz}-6.1 \mathrm{GHz}$ ).
\end{abstract}

Index Terms - Ultra wideband antennas, circular polarization, optimization, spline, slot antennas

\section{INTRODUCTION}

$\mathrm{W}$ ideband antenna research proliferated in the last decade due to the allocation of spectrum for ultra wideband (UWB) unlicensed use by various national regulators [1]. Many UWB antennas were developed and methodologies to measure antenna pulse distortion (e.g. group delay, fidelity factor) were established. However, these achievements mainly considered linearpolarization, leaving the development of wideband circularly-polarized (CP) systems behind.

Early theoretical investigations on ultrawideband CP systems were made by Shlivinski [2] in 2009 and followed by a practical implementation by Tan [3] in 2010, using an array with a complex feed network. However, unlike linearly-polarized antennas, the number of compact $\mathrm{CP}$ wideband designs is limited. Following this, a two-antenna array employing a wideband hybrid coupler was introduced realizing a $60 \% 3 \mathrm{~dB}$ axial-ratio (AR) bandwidth [4]. A CP array based on Vivaldi antennas was shown to offer 74\% AR bandwidth for two antennas [5] and $163 \%$ for four elements [6]. These approaches, despite having a wideband AR, are not practical for small terminal devices.

Solutions that are both compact and planar were reported in [7 - 16]. These include a dipole-like structure with $77 \%$ AR bandwidth [7]; a hexagonal slot antenna with 50\% AR bandwidth [8]; a radially symmetric aperture with 68\% AR bandwidth [9]; an L-shaped slot antenna providing 46.5\% AR bandwidth [10] or 32.2\% AR bandwidth and $132 \%$ impedance bandwidth for more complex geometries [11]. A combination of a slot and a monopole antenna is known to provide from 30\% [12] to 60\% [13] AR bandwidth, whereas a monopole with a parasitic stub yields $44.9 \%$ [14]. An inverted-F antenna in [15] was shown to provide $39.2 \%$ AR bandwidth whereas a spiral-inspired compact antenna exhibited 44.5\% [16].

This research on small UWB CP antennas dealt with complex geometries and a large number of antenna parameters. Typically the antennas consist of 8 to 17 optimizable parameters, whereas a parametric study is provided only for 1 to 4 key parameters [7, $8,10,12-14,16$ ]. It was shown in [10] that the effects of the ground plane on $S_{11}$ were either limited or predictable (i.e. frequency shifted downwards with increased dimension), however they have a significant impact on axial-ratio in a less predictable manner. In [14] an increase of ground plane height by only $0.5 \mathrm{~mm}$ from the optimal dimension decreased the CP bandwidth by $6 \%$. These studies show that compact UWB CP antennas are especially sensitive to changes in ground plane dimensions, posing a challenge for integration into commercial products, where the antenna usually must be modified (i.e. due to limited space, esthetic requirements or the need to incorporate other electronic components).

Manuscript received May 9, 2014. This work was supported by the Science Foundation Ireland under 13/TIDA/I2746, 10/CE/I1853 and by "ELEVATE: Irish Research Council International Career Development Fellowship - co-funded by Marie Curie Actions", grant no ELEVATE PD/2014/79.

A. Narbudowicz is with the Antenna \& High Frequency Research Centre, Dublin Institute of Technology and Institute of High Frequency Technology, RWTH Aachen University, 52056 Aachen, Germany (e-mail: adam.narbudowicz@ dit.ie).

M. John is with CTVR - the Telecommunication Research Centre, Trinity College Dublin, Dublin 2, Ireland.

X. L. Bao and M. J. Ammann are with the Antenna \& High Frequency Research Centre, Dublin Institute of Technology, Dublin 8, Ireland.

V. Sipal was with the Antenna \& High Frequency Research Centre, Dublin Institute of Technology, Dublin 8, Ireland. He is currently with the European Patent Office, the Hague, the Netherlands. 


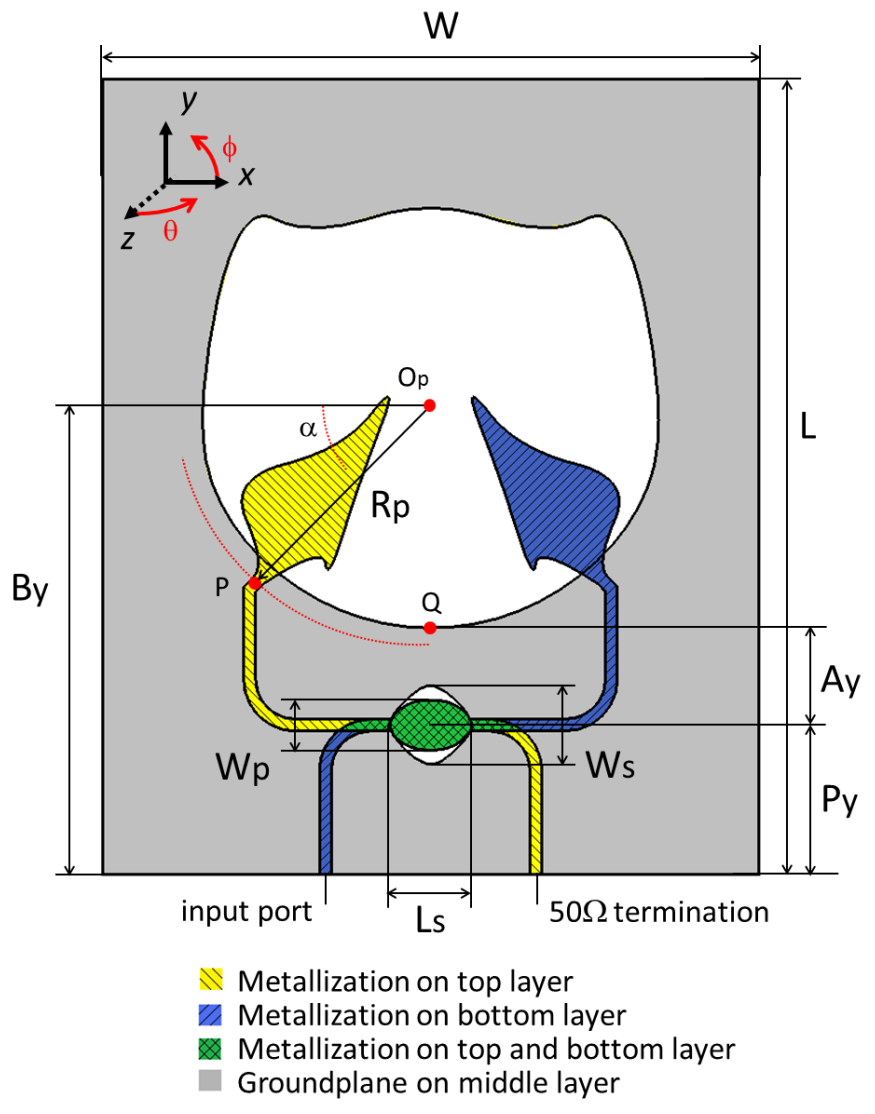

Fig. 1. Geometry of the proposed optimized UWB CP antenna.

The proposed work introduces a new UWB slot CP antenna with 54\% bandwidth and demonstrates an optimization technique, which, due to the use of spline curves, can quickly re-tune the antenna for integration into a target device. The antenna covers low duty cycle UWB band $(3.1-4.8 \mathrm{GHz})$ allowed in Europe for non-licensed use [1]. The paper demonstrates for the first time the use of spline curves for circularly-polarized antennas. This technique allows easy optimization, while preserving phase coherency required for good axial ratio across wide bandwidths. The optimization is based on a trust region framework algorithm [17] offered by CST Microwave Studio [18]. This algorithm is expected to provide quick convergence for cases, where initial parameters give a reasonable estimate of the optimum.

The proposed shape is a slot antenna with two orthogonal feed probes for CP excitation. The use of a slot allows confinement of the electric field and produces a bidirectional pattern. The probes establish two orthogonal electric field components. The use of spline curves allows smooth geometric transitions and preserves the feed signal phase coherence over wide bandwidth [19]. The planar feed probes are fed through a UWB phase shifter [20,21]. These phase shifters, first proposed by Abbosh and Bialkowski, are compact and offer stable $90^{\circ}$ phase shifts across very wide bandwidths.

\section{ANTENNA DESIGN}

Fig. 1 depicts the proposed antenna. The design consists of three layers of metallization, separated by two layers of Taconic ${ }^{\mathrm{TM}}$ RF-35 substrate (each of $0.5 \mathrm{~mm}$ thickness, $\varepsilon_{\mathrm{r}}=3.5, \delta_{\text {loss }}=0.0018$ ). To achieve a $90^{\circ}$ phase shift, a UWB phase shifter is designed according to [20] and incorporated into the antenna (seen in the lower part of Fig. 1). It consists of two microstrip lines, running parallel on opposite sides of the ground plane. Each microstrip line is loaded with an elliptical patch (with minor axis $W_{p}$ and major axis $L_{s}$ ) and the two patches are coupled through the elliptical slot in the ground plane (with minor axis $W_{s}$ and major axis $L_{s}$ ). The 
dimensions $W_{p}, L_{s}$ and $W_{s}$ were calculated, based on [20] and are: $W_{p}=5.1 \mathrm{~mm}, L_{s}=8.2 \mathrm{~mm}$ and $W_{s}=7.8 \mathrm{~mm}$.

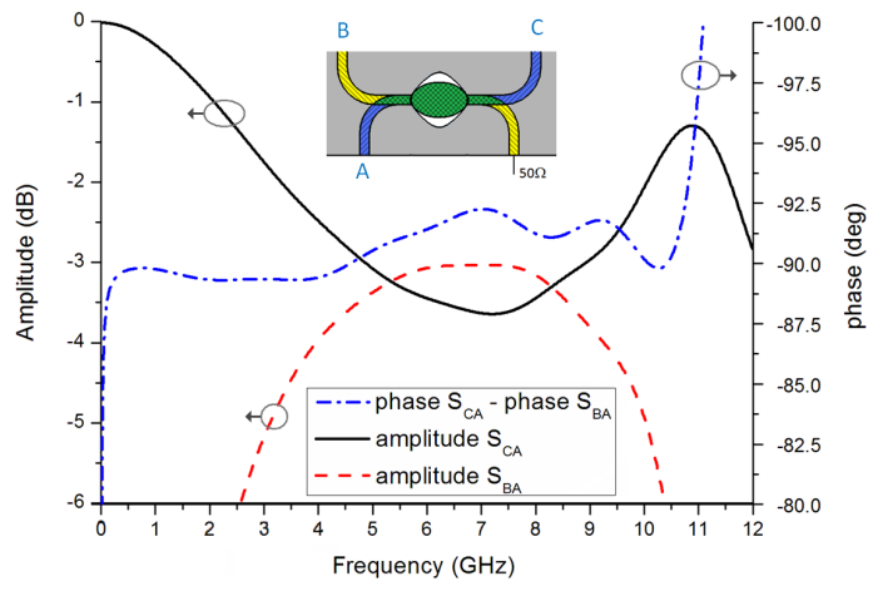

Fig. 2. Phase and amplitude of transmission coefficients of the prototyped phase shifter.

TABLE I

BOUNDARIES FOR CONTROL POINTS OPTIMIZATION (IN MM)

\begin{tabular}{ccc|cc}
\hline \multirow{3}{*}{ Control point } & \multicolumn{2}{c}{$u$ or $x$} & \multicolumn{2}{c}{$v$ or $y$} \\
\cline { 2 - 5 } & \multirow{2}{*}{$\min$} & $\max$ & $\min$ & $\max$ \\
\hline$M_{1}$ & 0 & 4 & 0 & 8.7 \\
$M_{2}$ & 1.3 & 10.7 & 0.6 & 16.7 \\
$M_{3}$ & -2.7 & 13.4 & 10.6 & 30 \\
$M_{4}$ & -6.7 & 2 & 6.6 & 30 \\
$M_{5}$ & -13.4 & 0.7 & 9.3 & 30 \\
$M_{6}$ & -12 & 1.3 & 1.3 & 16.7 \\
$M_{7}$ & -2.7 & 0 & 0 & 4.7 \\
$S_{1}$ & 12 & 28.1 & 10.6 & 45.5 \\
$S_{2}$ & 1.3 & 13.5 & 10.6 & 45.5 \\
$S_{3}$ & - & - & 10.6 & 45.5 \\
& & & &
\end{tabular}

An integrated phase shifter was used to provide greater robustness and ensure faster optimization convergence. Fig. 2 shows the simulated phase and amplitude reflection coefficients of the coupler. The second input port (unused by the antenna) is terminated with $50 \Omega$ to avoid reflections that would degrade the phase properties. The input port is denoted as A and the two output ports as $\mathrm{B}$ and C. A phase difference between ports $\mathrm{B}$ and $\mathrm{C}$ exhibit a stable $90^{\circ}$ performance $\left( \pm 3^{\circ}\right)$ in the range from $100 \mathrm{MHz}$ to 10.8 $\mathrm{GHz}$, as seen in Fig. 2. The transmission between ports $\mathrm{A}$ and $\mathrm{B}$ and between ports $\mathrm{A}$ and $\mathrm{C}$ provides a stable amplitude performance around $-3.5 \mathrm{~dB}( \pm 1.5 \mathrm{~dB})$ in the band from 3 to $9.3 \mathrm{GHz}$ and $\pm 0.5 \mathrm{~dB}$ in the band of 4.85 to $9.9 \mathrm{GHz}$. It can be seen, that the amplitude is the more significant limiting factor than the phase. The isolation between ports $\mathrm{B}$ and $\mathrm{C}$ is better than $30 \mathrm{~dB}$ in the range from $\mathrm{DC}$ to $8 \mathrm{GHz}$ and better than $20 \mathrm{~dB}$ for frequencies up to $10.3 \mathrm{GHz}$ (curve not shown for brevity).

Outputs B and C are connected via a $50 \Omega$ microstrip line to the feed probes, which extend over the slot in the ground plane. The two probes are initially oriented at an angle of $90^{\circ}$ with respect to each other, one on the top and one on the bottom metallization layer. This orientation is later optimized, where $\alpha$ (see Fig. 1) is allowed to vary from $0^{\circ}$ to $70^{\circ}$. The optimal position for the optimized antenna was found to be $\alpha=47.4^{\circ}$ (forming $85.2^{\circ}$ angle between optimized feed probes).

Locating feed probes on different layers simplifies the feed structure because no vias are required. However it also causes a $1 \mathrm{~mm}$ separation along the $z$ direction, corresponding to $9^{\circ}$ of phase shift at $4 \mathrm{GHz}$ (for $\varepsilon_{\mathrm{r}}=3.5$ ). This separation cannot be reduced by using a thinner substrate, as its thickness is dictated by the phase shifter coupling properties. As a consequence an asymmetry is seen between the front $(+\mathrm{z})$ and rear $(-\mathrm{z})$ directions. This is especially visible for the AR level and means that an improvement of $\mathrm{AR}$ on one side (e.g. $\theta=0^{\circ}$ ) would cause a degradation on the other side $\left(\right.$ e.g. $\left.\theta=180^{\circ}\right)$. Hence, the optimization goal was set only for the $+z$ direction; i.e. $\theta=0^{\circ}$.

The points marked in Fig. 1 as $P$ and $Q$ are the origins of the coordinate systems used in the optimization process to define spline curves. In order to optimize the angle $\alpha$ between the feed probes, point $P$ scribes an $\operatorname{arc}$ of radius $R_{p}$ with $O_{p}$ as origin. Antenna dimensions that remain constant throughout the optimization process are as follows: $W=66 \mathrm{~mm} ; L=80 \mathrm{~mm} ; A_{y}=8 \mathrm{~mm}$; 
$B_{y}=48 \mathrm{~mm} ; R_{p}=25.2 \mathrm{~mm} ; P_{y}=15 \mathrm{~mm} ; W_{s}=7.8 \mathrm{~mm} ; W_{p}=5.1 \mathrm{~mm} ; L_{s}=8.2 \mathrm{~mm}$.

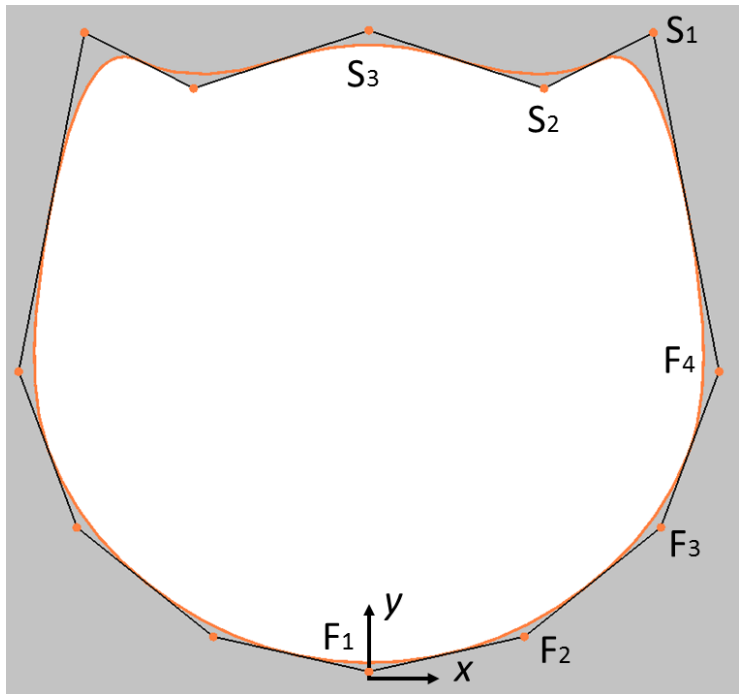

Fig. 3. Slot in the groundplane, as defined by a spline curve and its control points. The position of points $S_{1}-S_{3}$ was subjected to the optimization process, while points $F_{1}-F_{4}$ were fixed.

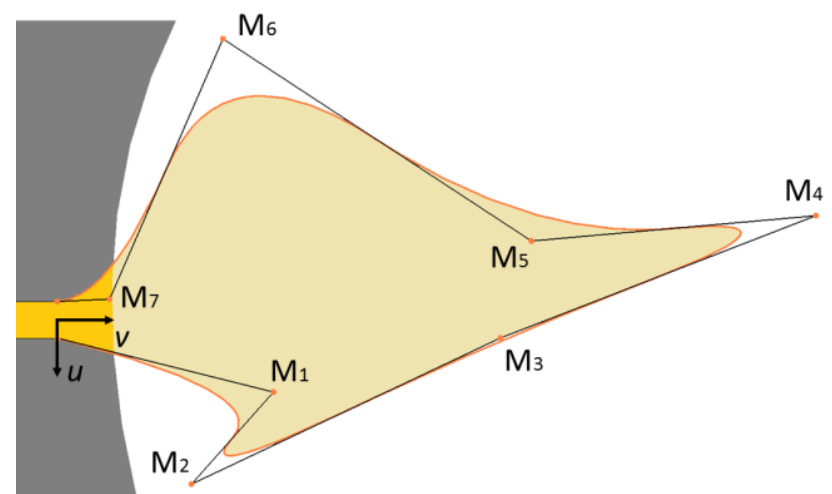

Fig. 4. A feed probe, as defined by a spline curve and its control points $M_{I^{-}}$ $M_{7}$. The second probe is symmetrical with respect to the $y$ axis. The coordinate system shown $(u, v)$ originates in point $P$.

\section{ANTENNA OPTIMIZATION}

The slot in the groundplane is defined by a single closed spline loop with its local coordinate system in point $Q$ (Fig 1 ). The bottom half of the curve (defined by control points $F_{1}-F_{4}$ ) is a semi-circle which is not part of the optimization. This is to facilitate the optimization of angle $\alpha$. The top half is symmetric and involves 3 control points $S_{1}-S_{3}$ with 5 moveable parameters for the optimizer, as shown in Fig. 3. Note point $S_{l}$ is allowed to move only along $y$ axis.

The feed probes are defined with respect to a local coordinate system in point $P$. This point scribes an arc with a constant radius of $R_{p}=25.2 \mathrm{~mm}$ and origin $O_{p}$ (i.e. around the semi-circle defined by points $F_{1}-F_{4}$ ). The angle of rotation $\alpha$ (seen in Fig. 1 ) is a parameter for optimization. Each feed probe is defined by 7 control points in this coordinate system (shown in Fig. 4 ), giving 14 parameters to be optimized.

A Trust Region Framework was used as an optimization algorithm. This algorithm is offered with the CST Studio Suite and is intended for localized optimization, where initial parameters give a reasonable estimation of the optimum performance [17]. This is considered to be the case with the proposed antenna, as the designed UWB phase shifter satisfies the basic phase requirement for CP. The slot and probes are optimized to ensure good radiation efficiency, with the phase centers of the two components close enough to produce good CP at boresight. It can also compensate for small inaccuracies of the phase shifter.

The antenna was optimized with a goal requiring AR $<3 \mathrm{~dB}$, total efficiency above $60 \%$ and $\mathrm{S} 11<-10 \mathrm{~dB}$ in the band of $3-8$ $\mathrm{GHz}(88 \%)$. These goals were weighted $60 \%, 30 \%$ and $10 \%$ respectively. The AR was evaluated for $\theta=0^{\circ}$ (i.e. $+z$ direction).

The optimization involves 9 spline control points moving in two dimensions, one spline control point moving in single dimension and the angle $\alpha$. This results in 20 optimization parameters. The parameters were allowed to move within the boundaries specified in Table I. For the slot (points $S_{1}-S_{3}$ ), the lower boundary in the $y$ direction is the "height" of point $F_{3}$ in order to prevent long, 
narrow slots. The upper limit is set to $45.5 \mathrm{~mm}$ to prevent the slot from breaking through the upper edge of the ground plane and forming an open slot. To preserve symmetry, the middle point $\mathrm{S}_{3}$ is fixed at $x=0$. The length (along $x$ ) between points $\mathrm{S}_{3}$ and $\mathrm{F}_{4}$ is divided into two areas, forming boundaries for points $S_{1}$ and $S_{2}$. However the upper limits of both those boundaries are slightly extended, to provide overlapping and to extend the $S_{1}$ boundary $4 \mathrm{~mm}$ beyond point $\mathrm{F}_{4}$.

For the probe optimization, the boundaries from Table I are visualized in Fig 5. The most critical point is $\mathrm{M}_{4}$, which dictates the effective length of the probe structure; therefore it can move within large range in the $v$ direction. To keep it central, only modifications up to $8.7 \mathrm{~mm}$ within the $u$ direction are allowed, with more flexibility on the outer side of the probe, where it has no possibility to interfere with the orthogonal probe. Points $\mathrm{M}_{1}$ and $\mathrm{M}_{7}$ form transitions between the fixed microstrip line and the probe, therefore their boundaries were strongly limited, especially along $u$. The minimum boundary for $\mathrm{M}_{3}$ was decreased to mitigate possible interference with the other probe.

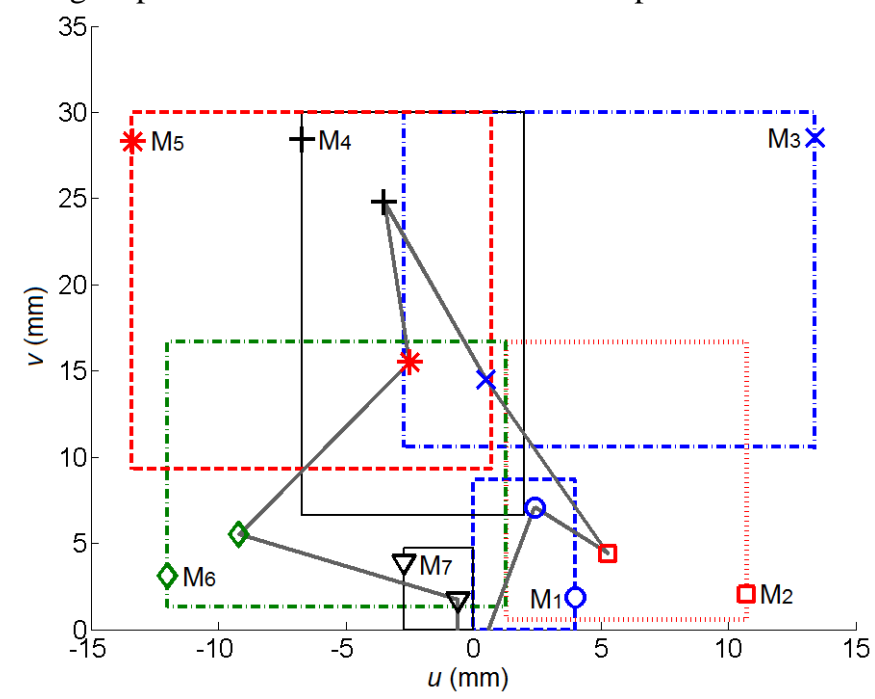

Fig. 5. Optimization boundaries for points $M_{1}-M_{7}$.

The solution was found after 129 runs of the time-domain solver, as shown in Fig. 6. This took approximately 144 hours on a Dell Precision T1500 (Intel core i7 CPU, 16GB RAM) workstation.

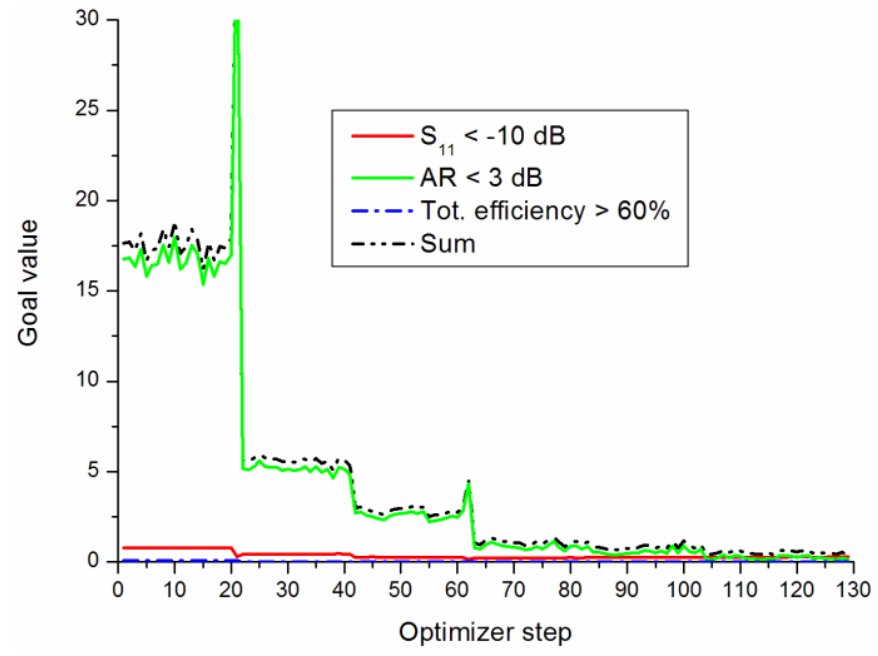

Fig. 6. Optimization goal function for consecutive steps of Trust Region Framework, as given by CST MWS.

The coordinates of the spline curve control points unequivocally determine the antenna shape. For the slot shown in Fig. 3, the coordinates are given in $\mathrm{mm}$ with respect to point $Q$ in the form of $S_{n}(x ; y)$. The optimized values are: $S_{1}=(19.5 ; 44.6) ; S_{2}=(12$; 40.8); $S_{3}=(0 ; 44.7)$. Other control points were not subjected to optimization. This was required to form a semi-circle, allowing point $P$ to move without obstruction for various angles $\alpha$. The coordinates of the fixed points are: $F_{1}=(0 ; 0.8) ; F_{2}=(10.7 ; 3.3)$; $F_{3}=(20 ; 10.7) ; F_{4}=(24 ; 21.4)$. Control points defining feed probes are described using a Cartesian coordinate system $(u, v)$. This system originates in point $P$ and is rotated with respect to $(x, y)$ coordinates by $\left(90^{\circ}-\alpha\right)=42.6^{\circ}$, as seen in Fig. 4. The optimized control points $M_{n}(u, v)$ are: $M_{1}=(2.4 ; 7.1) ; M_{2}=(5.3 ; 4.4) ; M_{3}=(0.5 ; 14.5) ; M_{4}=(-3.5 ; 24.8) ; M_{5}=(-2.5 ; 15.5) ; M_{6}=(-9.2 ; 5.5)$ and $M_{7}=(-0.6 ; 1.7)$. 


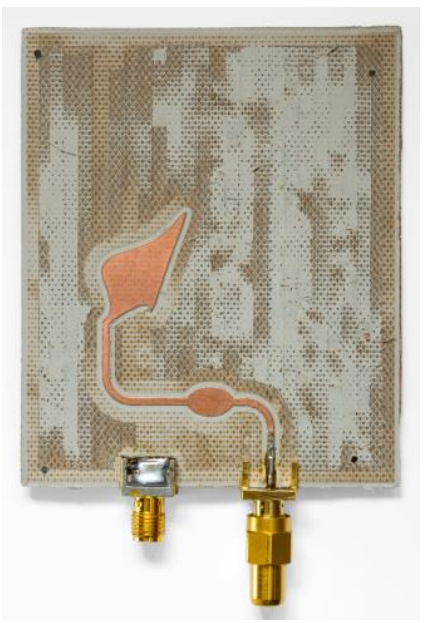

Fig. 7. Photograph of the optimized antenna.

\section{MEASUREMENTS}

The optimized UWB CP antenna was prototyped and measured, as depicted in Fig. 7. Fig. 8 compares the measured and simulated $S_{11}$ and Fig. 9 the AR in the $+z$ direction $\left(\theta=0^{\circ}\right)$. These were two parameters that formed the optimization goal. Figs. 8 and 9 show reasonable agreement between measurement and simulation. In simulation, the AR $\leq 3 \mathrm{~dB}$ from $3-8.8 \mathrm{GHz}$ and $\mathrm{S}_{11} \leq-10 \mathrm{~dB}$ from $2.1 \mathrm{GHz}$ to above $10.7 \mathrm{GHz}$ with minor peaks exceeding this limit by no more than $1.2 \mathrm{~dB}$. The measured AR $\leq 3 \mathrm{~dB}$ in the ranges from $3.2 \mathrm{GHz}$ to $6.1 \mathrm{GHz}(54 \%)$, with $\mathrm{S}_{11} \leq-10 \mathrm{~dB}$ largely exceeding this band $(2-12 \mathrm{GHz})$. The efficiencies are seen in Fig. 10. This is an important parameter as the antenna incorporates a lossy component (50 $\Omega$ match). This element causes the efficiency to become a function of the antenna design and therefore should be included in the optimisation process.

The simulated efficiency results are better than $58 \%$ across the $\mathrm{CP}$ bandwidth $(3.2-6.1 \mathrm{GHz})$, with a minimum at $5.8 \mathrm{GHz}$. The measured efficiency around $4.5 \mathrm{GHz}$ is lower than expected from the simulation, with a minimum of $51 \%$. This discrepancy is probably due to the cyanoacrylate glue, which was used to bond the structure. As seen in Fig. 11, it impacts both RHCP and LHCP, therefore the axial-ratio is still good at this frequency as seen in Fig. 9. The use of more advanced commercial manufacturing techniques are expected to increase the efficiency.

Around $6 \mathrm{GHz}$ a drop in total efficiency is seen, both in simulation and measurement. Despite this, the realized gain at $\theta=0^{\circ}$ is not strongly affected. This is because of a sharp peak of directivity, seen in Fig. 12.

Fig. 11 shows the measured and simulated RHCP and LHCP realized gain (for $\theta=0^{\circ}$ ) which are in reasonable agreement. In the CP band of $3.2-6.1 \mathrm{GHz}$ the RHCP peak gain is approximately $1.6 \mathrm{dBic}$ and is generally stable throughout this bandwidth (with some noise due to measurement facilities). Above $7 \mathrm{GHz}$ both measured and simulated realized gains drop abruptly, which is due to the antenna radiating energy in other directions as a consequence of the large electrical distance between two probes (as seen in Fig. 14e). The cross-polarization (LHCP) also shows reasonable agreement between measurement and simulation. Some discrepancy can be seen at $4-4.5 \mathrm{GHz}$, where the measured cross polarisation is lower than simulated. This peak roughly corresponds to the decreased measured efficiency, as seen in Fig. 10.

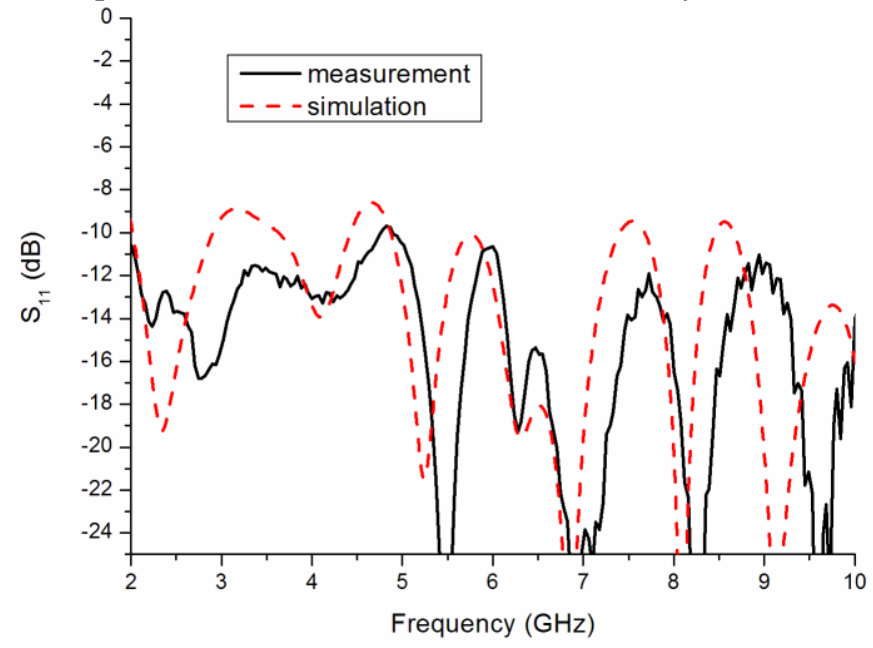

Fig. 8. Measured and simulated $\mathrm{S}_{11}$ of the proposed antenna. 


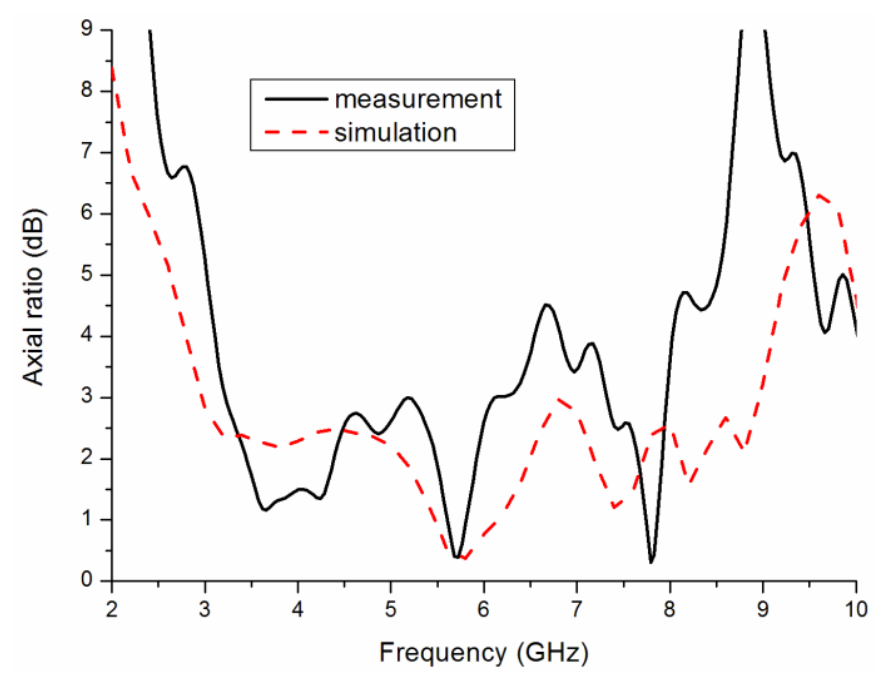

Fig. 9. Measured and simulated boresight axial-ratio of the proposed antenna.

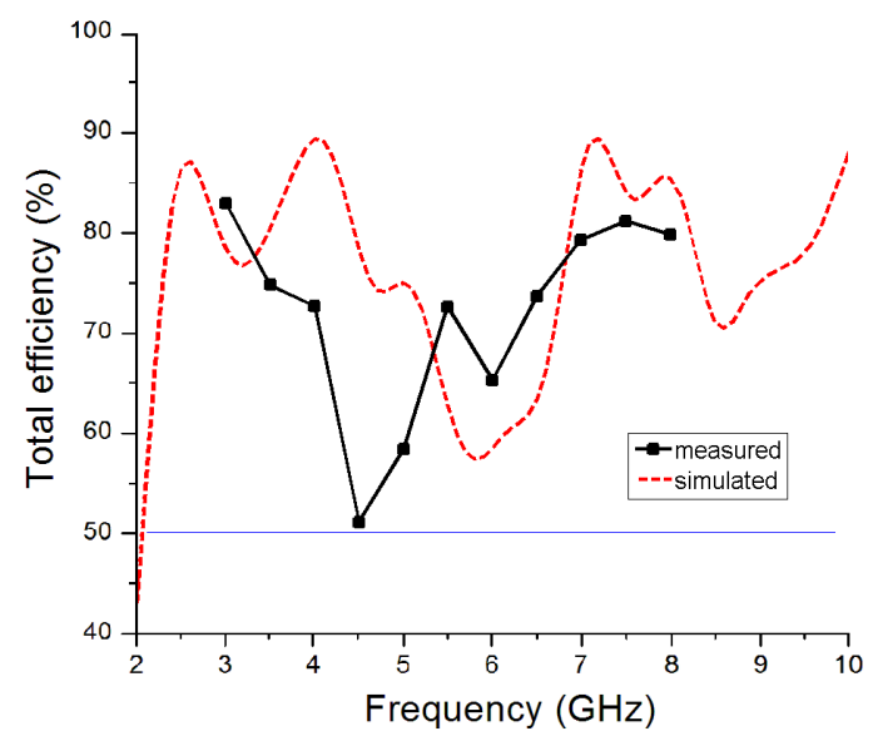

Fig. 10. Measured and simulated total efficiency.

Pulse dispersion is a significant phenomenon, limiting the performance of some UWB applications. The most common parameter to quantify its impact is fidelity factor. It describes the correlation between the shape of a pulse at antenna input and its shape at a given angle $(\theta, \phi)$ in the far-field [22]. This concept applies well to linearly polarized antennas. However it was demonstrated by Foltz et al. in [23] that a rotation of CP UWB antenna causes a constant phase-shift across all CP frequencies. This implies, that fidelity factor of $\mathrm{CP}$ antenna varies with antenna rotation, undermining the use of fidelity factor for $\mathrm{CP}$. To the best of our knowledge, there is no commonly accepted metric that allows the explicit quantification of pulse dispersion for CP. Therefore 


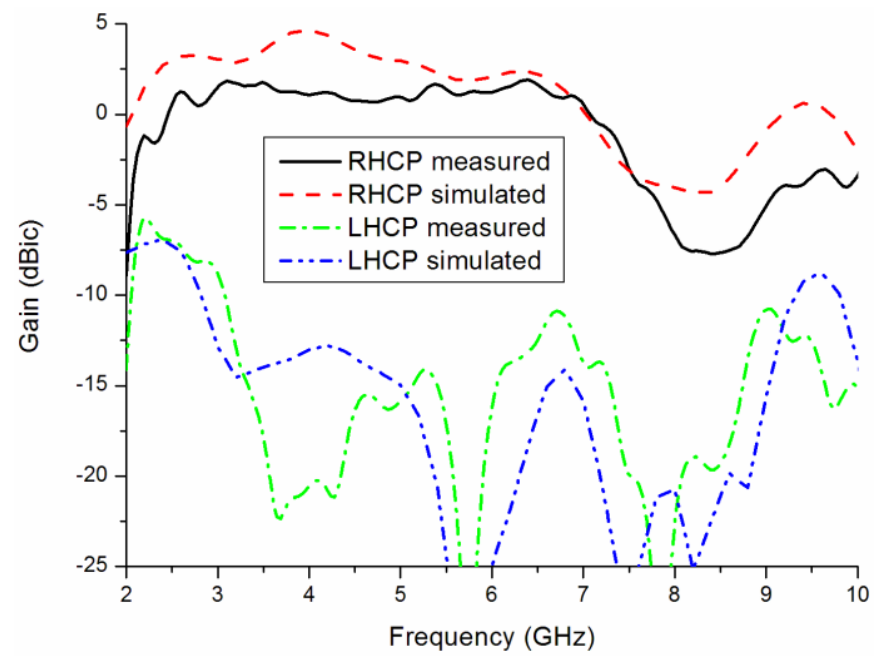

Fig. 11. Measured and simulated RHCP and LHCP gain at boresight.

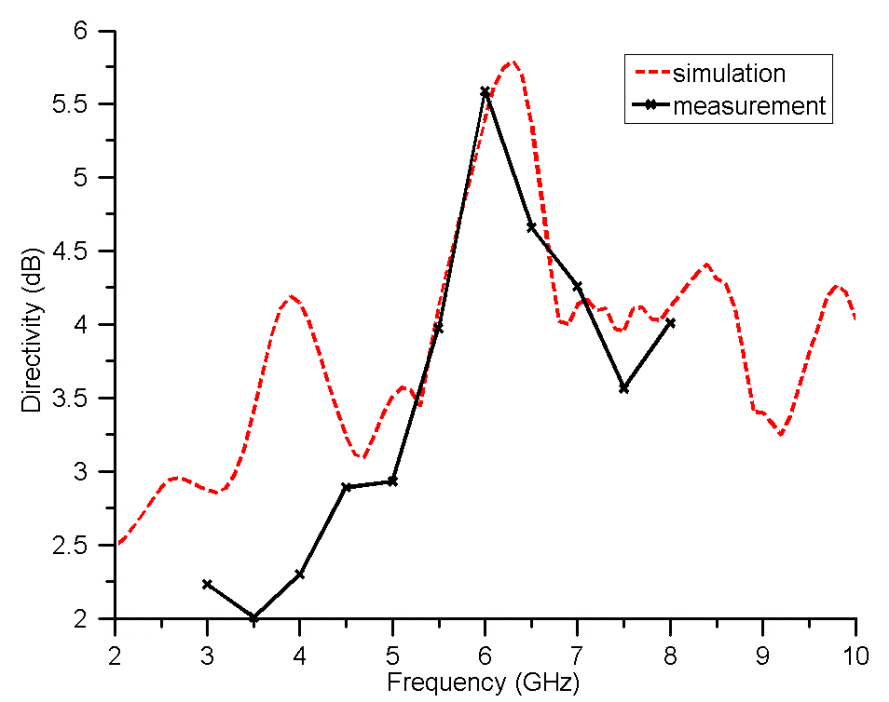

Fig. 12. Measured and simulated maximum directivity for RHCP.

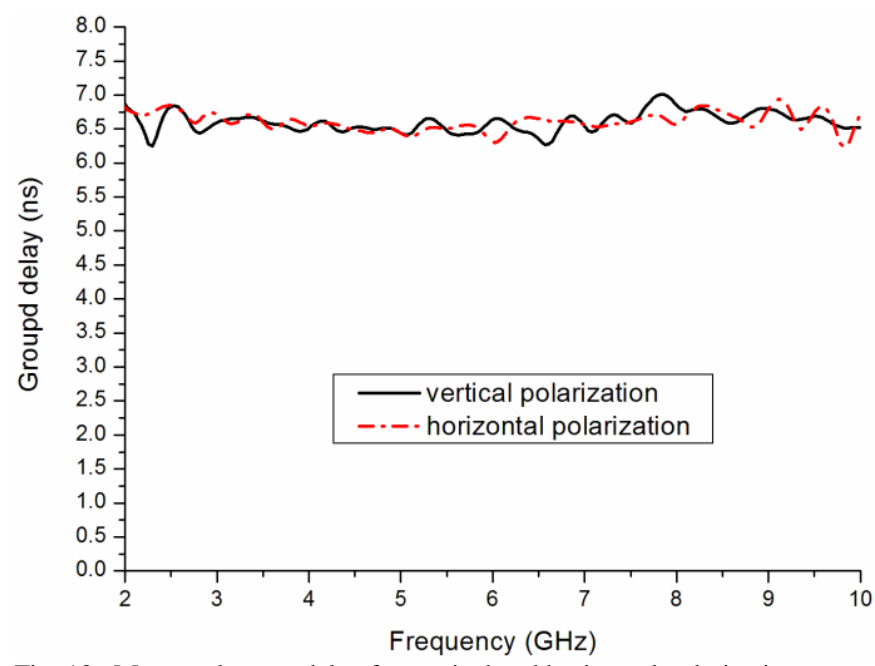

Fig. 13. Measured group delay for vertical and horizontal polarizations.

Fig. 13 shows the group delay for the $\theta=0^{\circ}$ direction for horizontal and vertical polarizations. The group delay was calculated directly from phase components of measured radiation patterns at boresight. A time-gating windows of $5 \mathrm{~ns}$ was applied to filter out possible reflections. It can be seen, that both polarizations have a flat group delay. 
Fig. 14 shows the realized gains of the antenna in $x z$-plane $\left(\phi=0^{\circ}\right)$. A good agreement between simulation and measurement is seen, demonstrating the usefulness of computer optimization for the proposed design. The two probes are spaced relatively close to one another, allowing a wide beamwidth. It can be seen, that the structure radiates RHCP in the $\theta=0^{\circ}$ direction and LHCP in the $\theta=180^{\circ}$ direction. This is expected for a slot antenna. However the optimization goal for the structure was set only with respect to the RHCP for the $+z$ direction $\left(\theta=0^{\circ}\right.$ in Fig. 14), therefore the LHCP beam presents a slightly worse AR performance and an asymmetry between the two can be seen. Neglecting the contribution of the phase shifter (i.e. assuming constant $3 \mathrm{~dB}$ power division and $90^{\circ}$ phase shift), the performance of the radiators is limited for lower frequencies by decreased efficiency and for upper frequencies by the main beam radiating off boresight due to larger electrical size.

\section{RE-OPTIMIZATION OF INTEGRATED ANTENNA}

To demonstrate the capabilities of the method, the proposed antenna was integrated into a typical terminal device. The device comprises of a solid copper plate oriented parallel to the antenna, and a PVC casing as shown in Fig. 15. The copper plate is the same size as antenna ( $66 \times 80 \times 1 \mathrm{~mm})$ and represents the populated device PCB. The PVC case is $1 \mathrm{~mm}$ thick with $\varepsilon_{r}=3$ and there is $0.2 \mathrm{~mm}$ airgap between the PCB and PVC. The copper plate was separated from the middle of the antenna by $\Delta_{\mathrm{m}}=19.5 \mathrm{~mm}$. This resulted in the distance between PVC walls (i.e. $\Delta_{\mathrm{m}}+4.3 \mathrm{~mm}$ ) being a quarter wavelength at the lowest operating frequency of $3 \mathrm{GHz}$. The overall size of the PVC casing is $68 \times 82 \times 27 \mathrm{~mm}$. The optimization used the original design as a starting point.

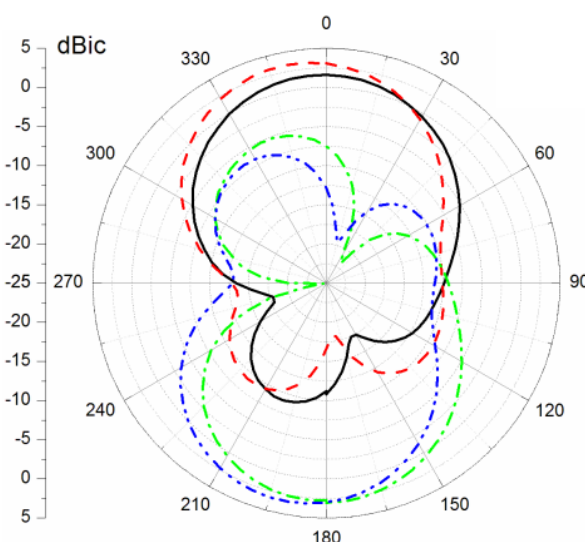

a)

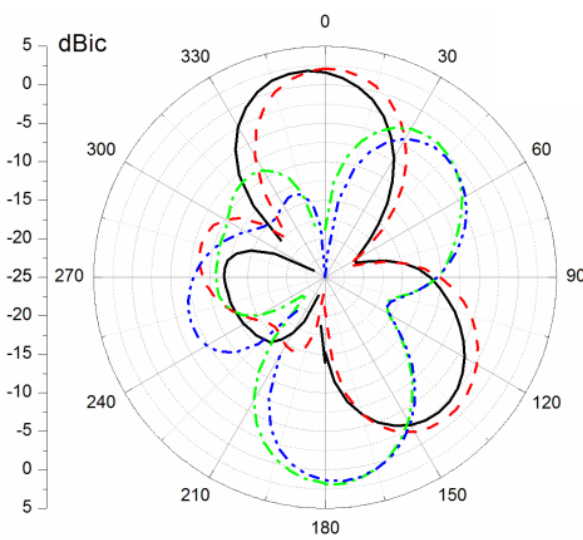

d)

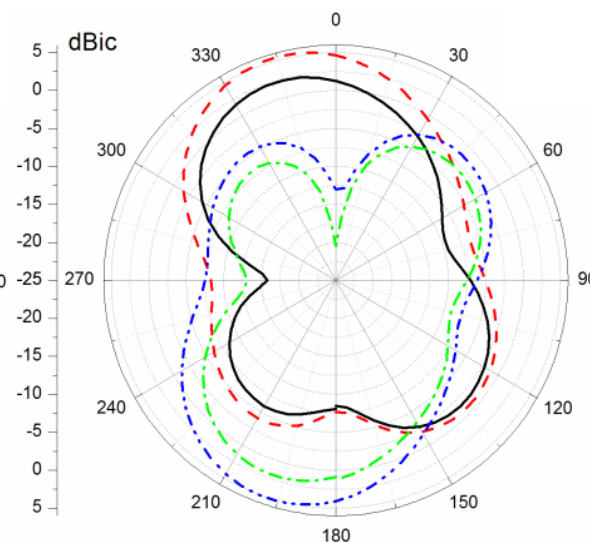

b)

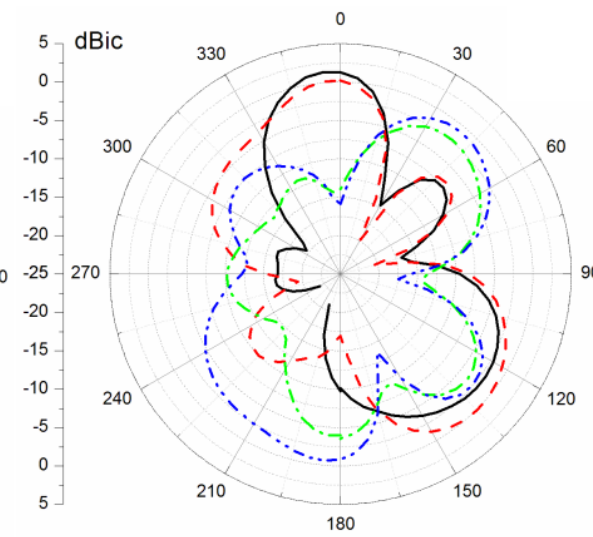

e)

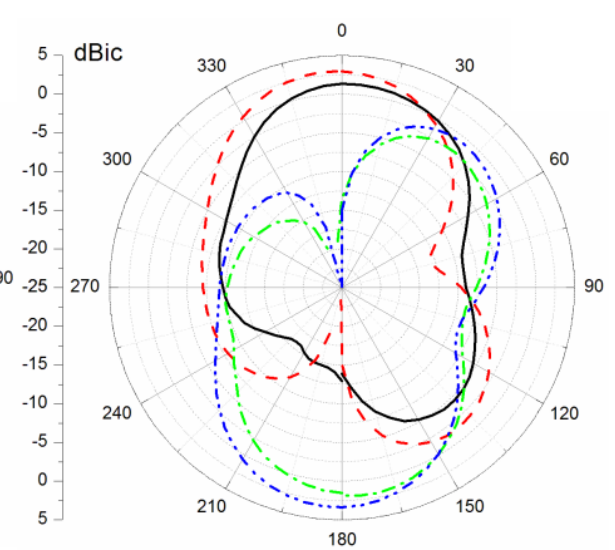

c)

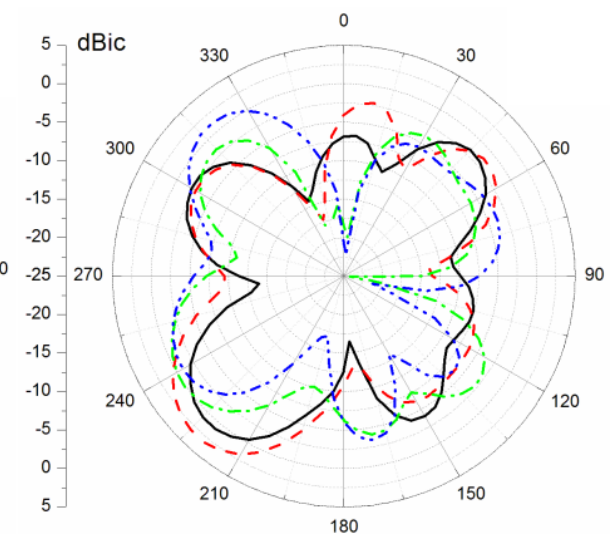

f)

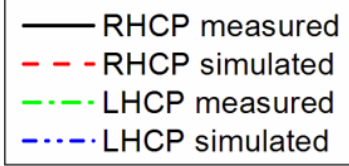

Fig. 14. Measured and simulated and realized gains in $x z$-plane: a) $3 \mathrm{GHz}$; b) $4 \mathrm{GHz}$; c) $5 \mathrm{GHz}$; d) $6 \mathrm{GHz}$; e) $7 \mathrm{GHz}$; f) $8 \mathrm{GHz}$. 


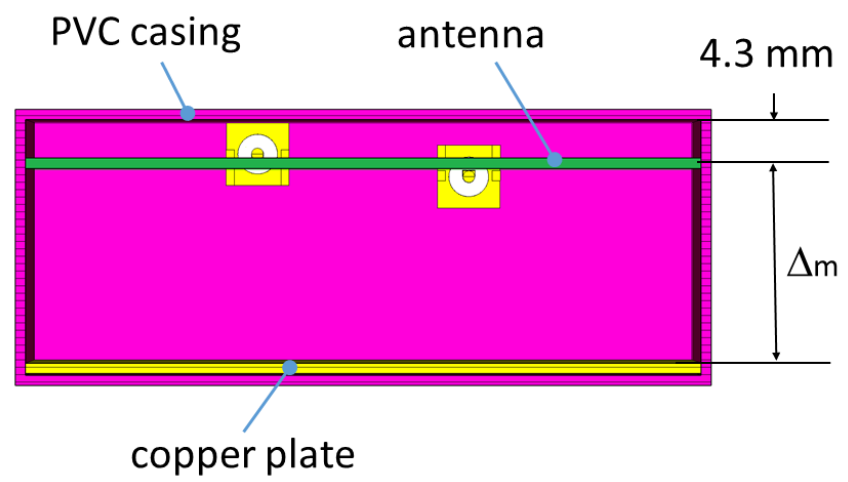

Fig. 15. Re-optimized antenna, embedded into typical terminal device (cut across the structure, as seen from $+y$ direction.

Fig. 16 shows a degradation of AR once the original antenna was integrated into the device. The AR can be seen degrading for higher frequencies, achieving $4.5 \mathrm{~dB}$ at $5.5 \mathrm{GHz}$. This is due to a resonance within the structure: at lower frequencies the distance between the antenna and the PCB is roughly one quarter wavelength, however for higher frequencies it increases towards a half wavelength, reinforcing negative interference and degrading the AR. It can be seen, that the optimization process was able to fully maintain the AR below the $3 \mathrm{~dB}$ within the whole optimization band. At the same time, almost no significant changes to $\mathrm{S}_{11}$ are visible.

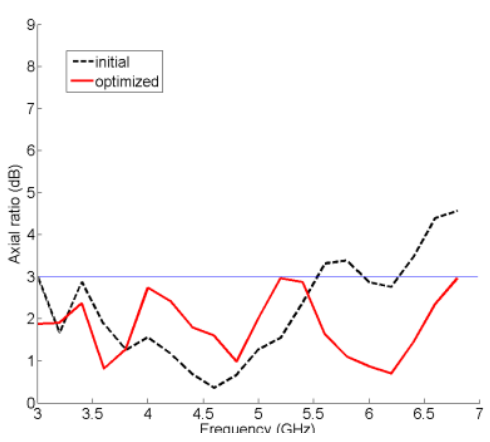

\section{OPTIMIZATION GUIDELINES}

To conclude the findings presented in this paper, brief guidelines for the design procedure are given:

1) The UWB phase shifter can be precisely designed for the required bandwidth using equations in [20]. This step will most likely determine substrate permittivity and thickness.

2) If the intended application is within a similar frequency range, the proposed antenna can be used as a starting point for optimization.

3) If the intended application requires a different frequency range, the design and boundaries for optimization (shown in Table I) should be scaled proportionally. For this purpose, $R_{p}$ should be kept around $1 / 4$ wavelength of the lowest operating frequency. This will determine the position of points $F_{1}-F_{4}$.

4) The boundaries for points $S_{1}-S_{2}$ should be kept between 0 and $F_{4}$ in the $x$ direction. For the $y$ direction the upper limit should ensure that the ground plane is at least $1 / 8$ wavelengths wide (at the lowest operating frequency).

\section{CONCLUSION}

The paper proposes a UWB CP antenna and an optimization procedure for such designs. The proposed antenna provides wideband CP performance in the $3.2 \mathrm{GHz}-6.1 \mathrm{GHz}(54 \%)$ band. The antenna has a good gain across all investigated frequencies and a flat group delay. It is planar and easy to manufacture with standard multilayered PCB technique.

The antenna performance is robust and allows for quick re-optimization, according to individual end-user requirements. For the investigated cases the convergence was reached within a reasonable 129 solver runs (within optimization space with 20 parameters). 


\section{ACKNOWLEDGMENT}

The authors would like to thank Prof. Dirk Heberling and Rasmus Cornelius from the Institute of High Frequency Technology, RWTH Aachen University for their help with antenna measurement.

\section{REFERENCES}

[1] V. Sipal, B. Allen, D. Edwards, and B. Honary, "Twenty years of ultrawideband: Opportunities and challenges," IET Communications, vol. 6, issue 10, pp. 1147-1162, Jul. 2012.

[2] A. Shlivinski, "Time-Domain Circularly Polarized Antennas," IEEE Trans. on Antennas and Propagation, vol. 57, issue 6, pp. 1606-1611, Jun. 2009.

[3] A. E.-C. Tan, M. Y.-W. Chia, and K. Rambabu, "Time Domain Characterization of Circularly Polarized Ultrawideband Array," IEEE Trans. on Antennas and Propagation, vol. 58, issue 11, pp. 3524-3531, Nov. 2010.

[4] X. Qing, Z. N. Chen, and H. L. Chung, "Ultra-wideband Circularly Polarized Wide-slot Antenna Fed by Three-stub Hybrid Coupler," in Proc. IEEE International Conference on Ultra-Wideband, Singapore, 2007, pp. 487-490.

[5] A. Narbudowicz, M. John, X. Bao and M. J. Ammann, "Vivaldi array for generation of UWB circular polarization," in Proc. Antennas and Propagation Society International Symposium, Chicago, USA, 2012, pp. 360.2.

[6] K.K.-M Chan, A.E.-C. Tan, and K. Rambabu, "Decade Bandwidth Circularly Polarized Antenna Array," IEEE Trans. on Antennas and Propagation, vol. 61, issue 11, pp. 5435-5443, Nov. 2013.

[7] K. G. Thomas, and G. Praveen, "A Novel Wideband Circularly Polarized Printed Antenna," IEEE Trans. on Antennas and Propagation, vol. 60, issue 12, pp. 5564-5570, Dec. 2012.

[8] S.-W. Zhou, P.-H. Li, Y. Wang, W.-H. Fend, and Z.-Q. Liu, "A CPW-Fed Broadband Circularly Polarized Regular-Hexagonal Slot Antenna With L-Shape Monopole," IEEE Antennas and Wireless Propagation Letters, vol. 10, pp. 1182-1185, Oct. 2011.

[9] Nasimuddin, Z. N. Chen, and X. Qing, "Symmetric-Aperture Antenna for Broadband Circular Polarization," IEEE Trans. on Antennas and Propagation, vol. 59, issue 10, pp. 3932-3936, Oct. 2011.

[10] S.-L. S. Yang, A. A. Kishk, and K.-F. Lee, "Wideband Circularly Polarized Antenna With L-Shaped Slot," IEEE Trans. on Antennas and Propagation, vol. 56, issue 6, pp. 1780-1783, Jun. 2008.

[11] J. Pourahmadazar, C. Ghobadi, J. Nourinia, N. Felegari, and H. Shirzad, "Broadband CPW-Fed Circularly Polarized Square Slot Antenna With Inverted-L Strips for UWB Applications," IEEE Antennas and Wireless Propagation Letters, vol. 10, pp. 369-372, May. 2011.

[12] T. Kumar, and A. R. Harish, "Broadband Circularly Polarized Printed Slot-Monopole Antenna," IEEE Antennas and Wireless Propagation Letters, vol. 12, pp. 1531-1534, Nov. 2013.

[13] T.-N. Chang, and J.-M. Lin, "Wideband circularly polarised antenna on slot-loaded ground plane," Electronics Letters, vol. 48, issue 14, pp. 818-819, Jul. 2012.

[14] L. Zhang, Y.-C. Jiao, Y. Ding, B. Chen, and Z.-B. Weng, "CPW-Fed Broadband Circularly Polarized Planar Monopole Antenna With Improved GroundPlane Structure," IEEE Trans. on Antennas and Propagation, vol. 61, issue 9, pp. 4824-4828, Sep. 2013.

[15] K. Hirabayashi, and M. Kominami, "Application of single point feeded wide band circularly polarized coplanar antenna," in Proc. International Symposium on Antennas and Propagation, Nagoya, Japan, 2012, pp. 579-582.

[16] Y. Shen, and C.L. Law, "A microstrip-fed quasi-spiral circularly polarized ultra-wideband antenna," in Proc. International Symposium on Antennas and Propagation, Spokane, USA, 2011, pp. 1463-1466.

[17] V. Sokol, "Optimization Techniques in CST STUDIO SUITE," in Proc. CST 6th European User Group Meeting, Munich, Germany, 2011, pp. 6-1-4 [online: http://www.cst.com/Content/Events/Downloads/eugm2011/Talk 6-1-4 CST UGM 2011.pdf].

[18] CST Microwave Studio - Workflow \& Solver Overview, CST - Computer Simulation Technology AG, Darmstadt, Germany, 2013.

[19] M. John, and M. J. Ammann, “Antenna Optimization With a Computationally Efficient Multiobjective Evolutionary Algorithm," IEEE Trans. on Antennas and Propagation, vol. 57, issue 1, pp. 260-263, Jan. 2009.

[20] A.M. Abbosh, and M.E. Bialkowski, "Design of Compact Directional Couplers for UWB Applications," IEEE Trans. on Microwave Theory and Techniques, vol. 55, issue 2, pp. 189-194, Feb. 2007.

[21] M. Sorn, R. Lech, and J. Mazur, "Simulation and Experiment of a Compact Wideband $90^{\circ}$ Differential Phase Shifter," IEEE Trans. on Microwave Theory and Techniques, vol. 60, issue 3, pp. 494-501, Mar. 2012.

[22] A. Dumoulin, M. John, M.J. Ammann, and P. McEvoy, "Optimized Monopole and Dipole Antennas for UWB Asset Tag Location Systems," IEEE Trans. on Antennas and Propagation, vol. 60, issue 6, pp. 2896-2904, June 2012.

[23] H. D. Foltz, J. S. McLean, A. Medina, and J. H. Alvarez Jerkov, "Lack of Rotation Invariance in Short-Pulse Communication Between Broadband CircularPolarization Antennas," IEEE Trans. on Antennas and Propagation, vol. 58, issue 11, pp. 3747-3751, Nov. 2010.

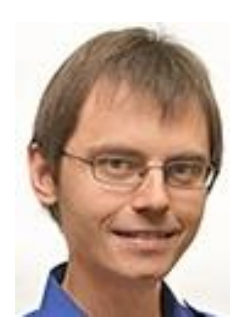

Adam Narbudowicz (S'12-M'14) received the M.Sc. degree from the Gdansk University of Technology, Gdansk, Poland in 2008, and the Ph.D. degree from the Dublin Institute of Technology, Dublin, Ireland, in 2013.

He is currently a Post-Doctoral Research Fellow funded by the Irish Research Council and Marie Cure Actions under the program "ELEVATE: Irish Research Council International Career Development Fellowship - cofunded by Marie Cure Actions". He works jointly at the Institute of High Frequency Technology, RWTH Aachen University, Aachen, Germany, and the Antenna and High Frequency Research Centre, Dublin Institute of Technology. He has published more than 20 scientific works in peer-reviewed journals and conferences, and chaired sessions on "Adaptive and reconfigurable antennas" and "MIMO Antennas" during EuCAP 2015 and APS/URSI 2015 respectively. His current research interests include switchless reconfigurable antennas, MIMO antennas and circular polarization.

Dr. Narbudowicz is a recipient of the 2012 DIT Inventor Competition Award for the best postgraduate/staff invention. 
Matthias John (M '07) received the degree (Dipl. Inf.) in computer science from the Deutsche Telekom University of Applied Science, Leipzig, Germany in 2004 and the Ph.D. in Microwave Antenna Engineering from the Dublin Institute of Technology, Dublin, Ireland in 2008.

$\mathrm{He}$ is currently working as a postdoctoral researcher in CTVR - the Telecommunications Research Centre in Trinity College Dublin. His research interests include UWB antennas, efficient numerical optimization techniques, antenna miniaturization, RFID and imaging applications. He is driving commercial activity in the center and received commercialization awards in 2009, 10 and 11 for technologies licensed to Irish and international companies. He also received a 2011 CST Award for work on "Miniature Ceramic Dual-PIFA Antenna to Support Band Group 1 UWB Functionality in Mobile Handset". He is funded by Science Foundation Ireland.

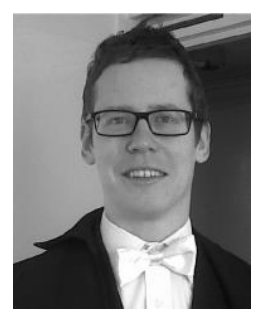

Vit Sipal (S'11-M'14) received the Bc. degree (B.Sc.) in Electrical Engineering from the Czech Technical University, Prague (Czech Republic) in 2006, the Dipl.-Ing. degree (MSc.) in Electrical Engineering from the RWTH Aachen University (Germany) in 2009, and the DPhil. degree in Engineering Science from the University of Oxford (U.K.) in 2012. Subsequently, he worked as the "Government of Ireland Postdoctoral Research Fellow in Engineering, Science and Technology" at the Dublin Institute of Technology. He is a holder of numerous awards for his academic and research achievements. He has published more than 40 scientific works in peer-reviewed journals and conferences, and acted as a reviewer for a number of international journals, conferences and for the Technology Agency of the Czech Republic. His research interests included antennas and wireless propagation for future communication systems, and the use of electromagnetic waves in sensor devices. He currently works as a patent examiner at the European Patent Office.

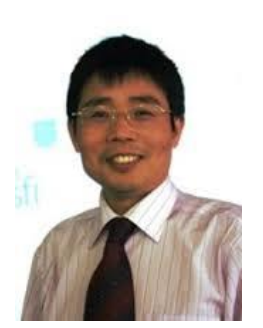

Xiulong Bao (M'09-SM'12) is a Research Fellow with the School of Electrical and Electronic Engineering, Dublin Institute of Technology, Ireland. He received the B.Sc. degree in physics from the Huaibei Normal University, Anhui Province, China in July 1991. He was awarded a M.Sc. in Physics and a Ph.D. in Electromagnetic Field and Microwave Technology from Southeast University, Jiangsu Province, China, in April 1996 and April 2003, respectively. After graduating, he was a Postdoctoral Researcher at Shanghai Jiaotong University, Shanghai, China, before going to Ireland in 2005. His broad research interests include analysis and design of various small and circularly polarized antennas, such as GPS antennas, multiple-band antennas, RFID antennas, a DTV antenna, handset antennas, Ultra Wideband (UWB) antennas and the design and application of metamaterial/EBG structures. He is also active in the study of electromagnetic scattering, electromagnetic numerical computation (FDTD, PSTD, FDFD and MOM methods) and the study of electromagnetic wave propagation and antenna theory. He has published 43 peer-reviewed journal papers and 40 conferences articles. He is an IEEE Senior Member and was Technical Program Committee member for the 65th IEEE Vehicular Technology Conference, Dublin, 2007.

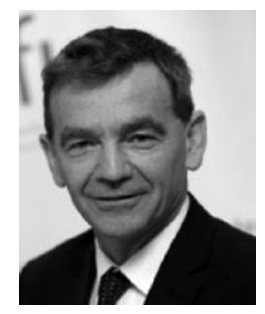

Max Ammann (M'96-SM'08) received the Council of Engineering Institution Part II degree in 1980 and the Ph.D. degree in microwave antenna design from Trinity College, University of Dublin, Ireland in 1997.

He is Assistant Head, School of Electrical and Electronic Engineering, Dublin Institute of Technology (DIT), where he is also the Director of the Antenna and High Frequency Research Centre. He spent eight years on radio systems engineering and antenna design for TCL/Philips Radio Communications Systems, Dublin, where he commissioned the Nationwide Communications Network for an Garda Siochana. In 1986 he joined the DIT as a Lecturer and was promoted to Senior Lecturer in 2003 and honorary professor in 2012. His research interests broadly include electromagnetic theory, antenna miniaturization for terminal and ultra wideband applications, antennas for medical devices and the integration with photovoltaic systems. He has in excess of 200 peer-reviewed papers published in journals and international conferences. He has served as an expert to industry on various antenna technologies in the communications, medical, aviation and electronic security sectors in Ireland and abroad. The roles have included design assessment, design solutions, technological strategy reporting and assessment of compliance with international standards on human exposure to electromagnetic energy. The industrial contacts also stem from several successful transfers of fundamental design research into applied solutions.

Dr. Ammann's team received various best paper awards at international conferences on Antennas and Propagation and several commercialization awards. They were also recipients of CST University Publication Awards in 2008, 2011 and 2014 for papers on "Wideband Reconfigurable Rolled Planar Monopole Antenna", "Miniature Ceramic Dual-PIFA Antenna to Support Band Group 1 UWB Functionality in Mobile Handset" and "Footwear Antennas for Body Area Telemetry" in IEEE Transactions on Antennas \& Propagation. He sits on the management committee of the EU COST Action IC1102, "Versatile, Integrated, and Signal-aware Technologies for Antennas (VISTA)" and is a member of the EurAAP working group on Small Antennas. As a member of the IEEE International Committee for Electromagnetic Safety, he participated in the revision of the IEEE Std. C95.1, 2005 standard for Safety Levels with Respect to Human Exposure to Radio Frequency Electromagnetic Fields, $3 \mathrm{kHz}$ to $300 \mathrm{GHz}$. He has chaired and organized special sessions on small antennas, UWB antennas and UWB Wireless Communication Systems at EuCAP and IEEE APS \& VTC. He was the local chair for the October 2008 EU COST IC0603 workshop and meeting in Dublin. $\mathrm{He}$ is currently associate editor for the IEEE Antennas \& Wireless Propagation Letters. 
